\title{
A novel method for measuring patients' adherence to insulin dosing guidelines: introducing indicators of adherence Massoud Toussi*1, Carine Choleau ${ }^{2,3}$, Gérard Reach², Michel Cahané3, Avner Bar-Hen ${ }^{1}$ and Alain Venot ${ }^{1}$
}

\author{
Address: ${ }^{1}$ Laboratoire d'Informatique Médicale et Bioinformatique (LIM\&BIO EA 3969), UFR SMBH, Université Paris 13, Bobigny, France, ${ }^{2}$ Service \\ d'Endocrinologie, Diabétologie et Maladies Métaboliques, Hôpital Avicenne, Assistance publique-Hôpitaux de Paris, et EA 3412, CRNH-IdF, \\ Université Paris 13, Bobigny, France and ${ }^{3}$ Association Aide aux Jeunes Diabétiques, Paris, France \\ Email: Massoud Toussi* - massoudtoussi@gmail.com; Carine Choleau - carine.choleau@ajd-educ.org; Gérard Reach - gerard.reach@avc.aphp.fr; \\ Michel Cahané - michel.cahane@ajd-educ.org; Avner Bar-Hen - Avner.Bar-Hen@math-info.univ-paris5.fr; Alain Venot - alain.venot@univ- \\ paris13.fr \\ * Corresponding author
}

Published: 5 December 2008

BMC Medical Informatics and Decision Making 2008, 8:55 doi:10.1 186/1472-6947-8-55
Received: 17 June 2008

Accepted: 5 December 2008

This article is available from: http://www.biomedcentral.com/1472-6947/8/55

(C) 2008 Toussi et al; licensee BioMed Central Ltd.

This is an Open Access article distributed under the terms of the Creative Commons Attribution License (http://creativecommons.org/licenses/by/2.0), which permits unrestricted use, distribution, and reproduction in any medium, provided the original work is properly cited.

\begin{abstract}
Background: Diabetic type I patients are often advised to use dose adjustment guidelines to calculate their doses of insulin. Conventional methods of measuring patients' adherence are not applicable to these cases, because insulin doses are not determined in advance. We propose a method and a number of indicators to measure patients' conformance to these insulin dosing guidelines.

Methods: We used a database of logbooks of type I diabetic patients who participated in a summer camp. Patients used a guideline to calculate the doses of insulin lispro and glargine four times a day, and registered their injected doses in the database. We implemented the guideline in a computer system to calculate recommended doses. We then compared injected and recommended doses by using five indicators that we designed for this purpose: absolute agreement (AA): the two doses are the same; relative agreement (RA): there is a slight difference between them; extreme disagreement (ED): the administered and recommended doses are merely opposite; Under-treatment (UT) and over-treatment (OT): the injected dose is not enough or too high, respectively. We used weighted linear regression model to study the evolution of these indicators over time.

Results: We analyzed 1656 insulin doses injected by 28 patients during a three weeks camp. Overall indicator rates were $A A=45 \%, R A=30 \%, E D=2 \%, U T=26 \%$ and OT $=30 \%$. The highest rate of absolute agreement is obtained for insulin glargine $(A A=70 \%)$. One patient with alarming behavior ( $A A=29 \%, R A$ $=24 \%$ and $E D=8 \%$ ) was detected. The monitoring of these indicators over time revealed a crescendo curve of adherence rate which fitted well in a weighted linear model (slope $=0.85$, significance $=0.002$ ). This shows an improvement in the quality of therapeutic decision-making of patients during the camp.

Conclusion: Our method allowed the measurement of patients' adherence to their insulin adjustment guidelines. The indicators that we introduced were capable of providing quantitative data on the quality of patients' decision-making for the studied population as a whole, for each individual patient, for all injections, and for each time of injection separately. They can be implemented in monitoring systems to detect non-adherent patients.
\end{abstract}




\section{Background}

In some diseases such as diabetes, patients manage their own therapy. Insulin dependent diabetic patients are often advised to use dose adjustment guidelines to calculate their doses of insulin for each self-administration. However, they may follow their guidelines totally, partially, or not at all. The adherence of patients to these guidelines is very important and can affect their blood glucose levels.

Methods for measuring patient adherence to drug therapy include self-reporting [1], patient interviews [2], pill counting [3], exploration of pharmacy records [4], measuring of active drug metabolites in the blood [5], and using electronic monitoring devices [6]. However, these methods are not applicable for the evaluation of patients' adherence to their insulin adjustment guidelines, because these guidelines do not determine correct insulin doses in advance. In fact, dose adjustment guidelines are distinct from common prescriptions by giving the patients the role of therapeutic decision-makers.

Diabetes is a major worldwide problem; and non-adherence to antidiabetic therapy and its impacts are well-documented [7]. Concerning insulin adjustment, the new technology allows the monitoring of patients through telecare systems, which store capillary blood glucose (CBG) and insulin doses [8]. Some systems even allow predicting of blood glucose based on lifestyle and therapeutic variables [9]. Although these systems have invaluable role in providing decision support and improving the quality of blood glucose control, they do not allow measuring the patients' conformance to insulin adjustment guidelines.

We present a method as well as a number of indicators that we developed for analyzing and monitoring of patients' adherence to their dose adjustment guidelines. We demonstrate our method in a group of type 1 diabetic patients who used an insulin dose adjustment guideline during supervised summer camps. We discuss the results and explain how our method and especially our indicators can be used in monitoring systems for real-time surveillance.

\section{Methods}

\section{Patients}

We used a database of logbooks of patients attending summer camps organized by Aide aux Jeunes Diabétiques (AJD). The latter is a non-profit organization with a major role in diabetes education in France. Eligible patients were those aged between 13 and 17, whose parents accepted their participation in the study. Non-eligible patients were those who did not manage their diabetes on their own, and those with a coexisting disease.
Twenty-eight patients (16 males and 12 females) were included in the study. They used a guideline to calculate the doses of their insulin injections four times a day. All patients had the same diet and the same kind of physical activity during the camp. Their daily carbohydrate intake was constant and fixed in advance for each meal by AJD nutritionists. The camp personnel supervised patients to assure their good dietary compliance. Physicians registered patients' CBGs, self-administered insulin doses, physical activities, and any event on their health status in an online database (the database is accessible to patients and their physicians under authentication [10]). The French Consultative Committee for Protection of Persons in Biomedical Research (CCPPRB) approved the research protocol and the guideline. The de-identified patient dataset is accessible as add-on material to this article. (Additional file 1).

\section{The guideline}

We used the official AJD guideline, taught to patients during summer camp. It consists of two pages of simple recommendations for the adjustment of insulin dose in a basal-bolus schema of insulin therapy including one injection of insulin lispro (or insulin aspart) before each meal, and one injection of insulin glargine before going to bed. This guideline was not specific for the kind of insulin, but for the schema of basal-bolus insulin therapy.

The guideline made use of patient's CBGs, injected doses of insulin, any advent of hypo- or hyperglycemia, and physical activity in a rule based algorithm composed of conditions and actions. It did not take into account carbohydrate intake because it was considered constant for each meal (breakfast, lunch, snack, and dinner) during the camp. As an example, to adjust the dose of insulin lispro before breakfast, the guideline proposed the patient to consider the CBG results obtained before lunch for two previous days, CBG measured at the moment of injection, and any physical activity over the next few hours. The guideline did not indicate the absolute dose of insulin. Instead, it provided instructions for the patients to adjust their own ongoing dose. The guideline is available as addon material to this article. (Additional file 1).

\section{Computer methods}

We extracted the rules of the guideline manually, and implemented them in a computer system, written in $\mathrm{R}$ programming language version 2.6.1 [11]. Our system uses data from patient logbooks to generate a dose adjustment for each insulin injection. It then compares these computer-generated dose adjustments with patient selfadministered doses (figure 1). The system source code is available as add-on material to this article. 


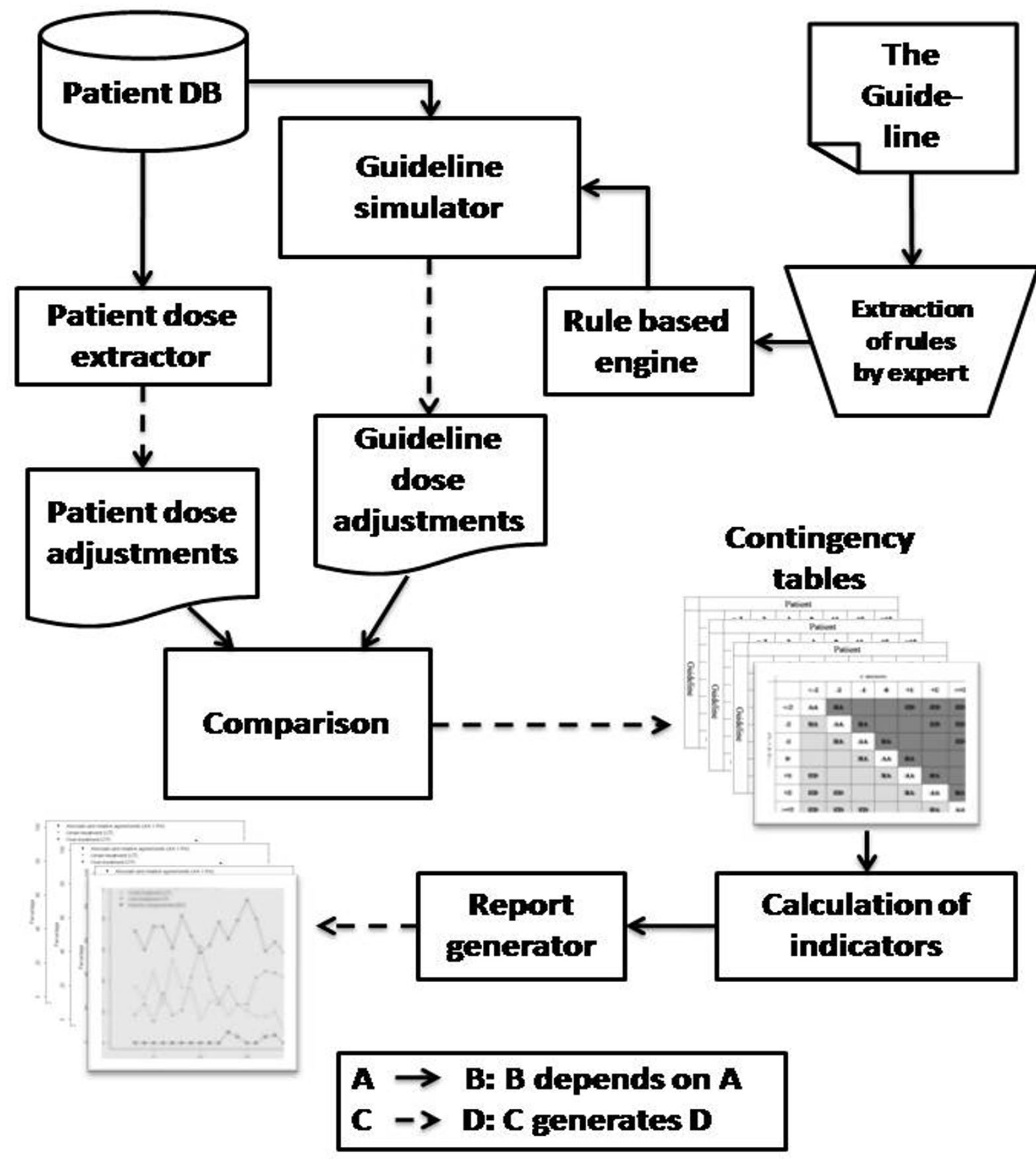

Figure I

System architecture.

\section{Designing indicators}

In order to be able to formalize and quantify the adherence, we categorized dose adjustments into seven discrete groups (table 1). We described each dose adjustment by variations with regard to the ongoing dose. By placing patient dose adjustments in columns and calculated dose adjustments in rows of a contingency table, we defined five indicators for quantifying patient's adherence to the guideline (figure 2). 
Table I: Dose adjustment categories.

\begin{tabular}{cl}
\hline Symbol & Meaning \\
\hline$<-2$ & Decrease dose by more than 2 units \\
\hline-2 & Decrease dose by between I and 2 units \\
\hline- I & Decrease dose by between 0 and I units \\
\hline $\mathbf{0}$ & Do not change the dose \\
\hline$+\mathbf{I}$ & Increase dose by between 0 and I units \\
\hline+2 & Increase dose by between I and 2 units \\
\hline$>+2$ & Increase dose by more than 2 units \\
\hline
\end{tabular}

a) Absolute agreement (AA): On-diagonal cell counts, which correspond to injections for which the patient's dose adjustment is the same as generated dose adjustment.

b) Under-treatment (UT): Off-diagonal cell counts situated below the diagonal, which correspond to injections for which patient's dose adjustment is smaller than generated dose adjustment. Clinically, this indicator represents a hypoglycemia avoiding behavior.

c) Over-treatment (OT): Off-diagonal cell counts situated above the diagonal, which correspond to injections for which patient's dose adjustment is greater than generated dose adjustment. Clinically, this indicator represents a hyperglycemia avoiding behavior.

d) Relative agreement (RA): Off-diagonal cell counts adjacent to the diagonal, which correspond to adherent cases, but with some degree of tolerance.

e) Extreme disagreement (ED): The outermost off-diagonal cell counts, which correspond to "potentially dangerous" cases. In our study, we defined this area as cell counts where patient dose adjustment was in an opposite direction (i.e. increase versus decrease) of generated dose adjustment.

\section{Statistical methods}

We used normal approximation of binomial distribution to construct confidence intervals (CI) for each indicator. We used also a weighted linear regression model [12] to determine the relationship between the time passed in the camp and each indicator. In the weighted analysis, we used the value of standard deviation of the indicator as the weight. Calculations are made by glm( ) function of R programming language version 2.6.1.

\section{Results}

Patients mean age was $15(\mathrm{SD}=1)$ years and their mean body mass index was $20.3(\mathrm{SD}=2.8) \mathrm{kg} / \mathrm{m}^{2}$. Mean $\mathrm{HbA}_{1 \mathrm{C}}$ was $7.8(\mathrm{SD}=1.2) \%$ at the beginning of the study. Mean CBG was $130(\mathrm{SD}=70) \mathrm{mg} / \mathrm{dl}$ before breakfast, 170 (SD = $90) \mathrm{mg} / \mathrm{dl}$ before lunch, $180(\mathrm{SD}=90) \mathrm{mg} / \mathrm{dl}$ before dinner, and $170(S D=90) \mathrm{mg} / \mathrm{dl}$ at bedtime.

Mean injected daily doses of insulin lispro were 9.5 (SD = 4.4) units before breakfast, $9.4(\mathrm{SD}=5.3)$ units before lunch, and $8.6(\mathrm{SD}=4.1)$ units before dinner. Mean injected daily dose of insulin glargine was $20.7(S D=7.2)$ units at bedtime.

Physicians made dose adjustments for patients on the first and the last two days of the camp. On all other days, patients adjusted themselves their doses of insulin under the supervision of physicians. Mean percentages of completion of the online database for dose adjustments before breakfast, lunch, dinner and at bedtime were respectively $92.6(\mathrm{SD}=3.8), 90.4(\mathrm{SD}=5.4), 86.7(\mathrm{SD}=4.6)$ and 86.9 $(\mathrm{SD}=4.8)$.

\section{Overall analysis}

We analyzed 1656 pairs of dose adjustments (table 2). Two adjustments "do not change dose" and "decrease dose by up to 1 unit" were the most common adjustments in both patient (51\% and 15\% respectively) and computer-generated dose adjustments (62\% and 20\%). While guideline rules did not allow the dose to be adjusted by more than 2 units, patients made adjustments by more than 2 units in $7 \%$ of injections.

Overall rates of indicators for all patients and all insulin types were $\mathrm{AA}=45 \%(\mathrm{CI}=2.3 \%), \mathrm{RA}=30 \%(\mathrm{CI}=2.1), \mathrm{ED}$ $=2 \%, \mathrm{UT}=26 \%(\mathrm{CI}=2.3)$ and $\mathrm{OT}=30 \%(\mathrm{CI}=2.1)$. As $\mathrm{ED}$ was too close to zero, its confidence interval was not calculated.

Although over-treatment (OT) was more common than under-treatment (UT), there were less cell counts of extreme disagreement (ED) in OT area than in UT area (10 and 16 cases respectively). This suggests that extreme disagreement was more likely to be a result of under-treatment, i.e. hypoglycemia-avoiding behavior.

\section{Injection related analysis}

We calculated indicators separately for different injections (table 3). Patients were mostly adherent concerning insulin glargine with a rate of $\mathrm{AA}=70 \%$ followed by insulin lispro before breakfast $(\mathrm{AA}=38 \%)$. By considering RA cells also as adherent (with some tolerance), insulin lispro injections before dinner represented the highest level of adherence (78\%). 


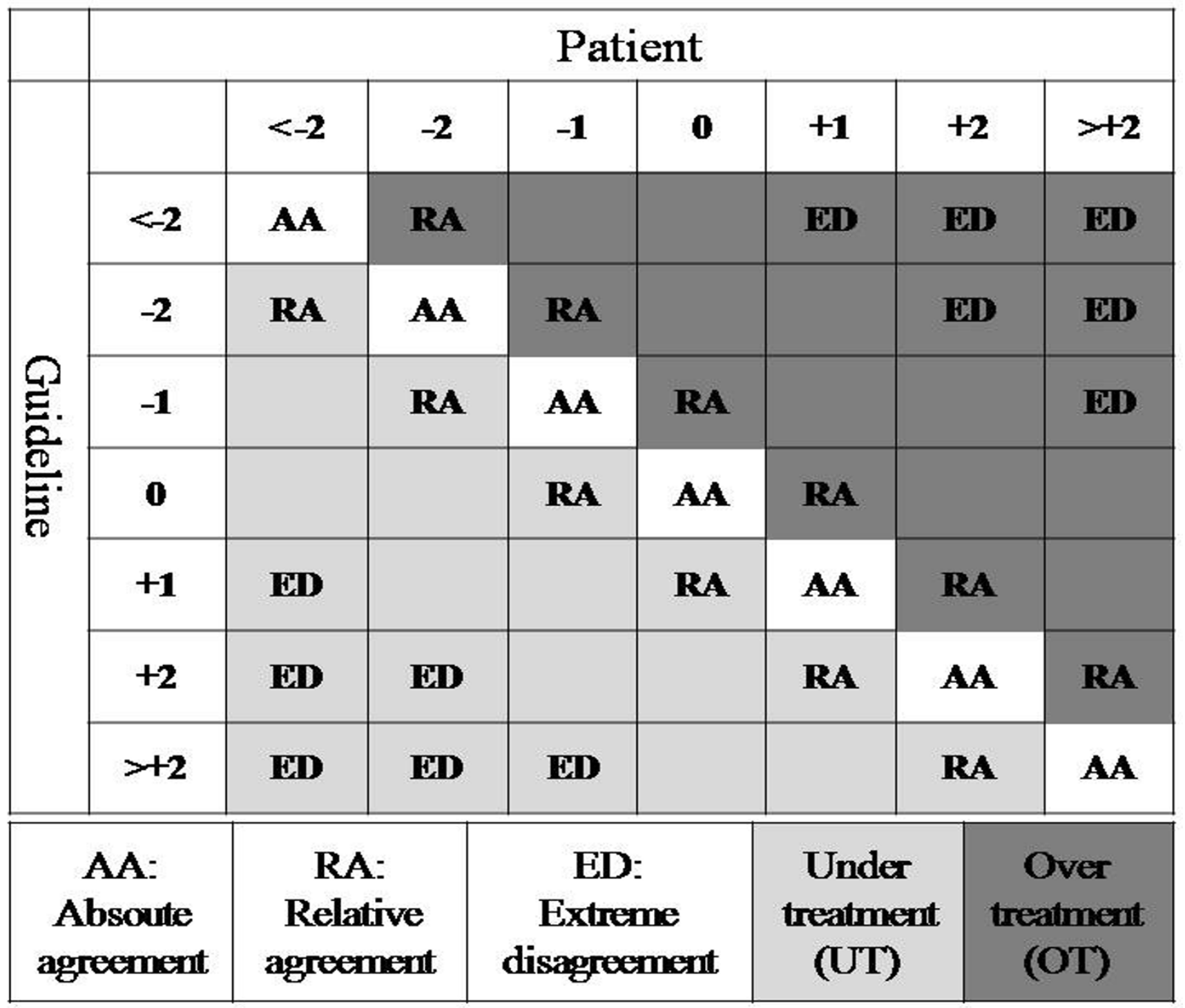

Figure 2

Indicators of adherence.

\section{Patient related analysis}

We calculated also the indicators for each patient (table 4 ). The highest score of adherence to the guideline was observed in a patient with AA plus RA ratio of $96 \%$. On the other side, the highest rate of alarming non adherence -marked by the highest rate of ED indicator- was detected in a patient with an $\mathrm{ED}$ rate of $8 \%$.

\section{Time related analysis}

We analyzed the evolution of patients' therapeutic decisions over time by tracing the indicators of each day separately (figure 3). The AA plus RA curve, which was more interesting for clinicians than AA or RA alone, showed an increasing trend during the camp. The ED curve showed fluctuations apparently due to different weekend activities and feasts (non-adherence to the guideline increased during festivities). OT and UT curves showed that over-treatment was more common than under-treatment, almost all time.

We fitted a weighted generalized linear model to examine the evolution of each indicator. The model fitted well (significance $=0.002$ ) for the AA plus RA curve, but not for other indicators. It showed that the overall agreement progressed during the camp, and the rates of over- and undertreatments decreased smoothly. The extreme disagreement indicator, however, did not show a sensible change. 
Table 2: Patients' versus computer-generated dose adjustments.

\begin{tabular}{|c|c|c|c|c|c|c|c|c|c|}
\hline & & \multicolumn{7}{|c|}{ PATIENT DOSE ADJUSTMENTS } & \multirow[b]{2}{*}{ Total } \\
\hline & & $<-2$ & -2 & -1 & $\mathbf{0}$ & $\mathbf{I}$ & 2 & $>+2$ & \\
\hline \multirow[t]{8}{*}{ GUIDELINE DOSE ADJUSTMENTS } & $<-2$ & 0 & I & 0 & 3 & 0 & 0 & 0 & 4 \\
\hline & -2 & 3 & 8 & 4 & 49 & 4 & 6 & 0 & 74 \\
\hline & -1 & 13 & 37 & 75 & 125 & 49 & 23 & 4 & 326 \\
\hline & 0 & 31 & 69 & 143 & 609 & 101 & 51 & 28 & 1032 \\
\hline & I & 12 & 14 & 23 & 49 & 46 & 29 & 15 & 188 \\
\hline & 2 & 2 & 2 & 3 & 18 & 5 & 2 & 0 & 32 \\
\hline & $>+2$ & 0 & 0 & 0 & 0 & 0 & 0 & 0 & 0 \\
\hline & Total & 61 & $13 \mid$ & 248 & 853 & 205 & 111 & 47 & 1656 \\
\hline
\end{tabular}

\section{Discussion}

We presented a method for measuring and analyzing patients' adherence to an insulin dose adjustment guideline. A computer system deployed the guideline, and generated dose adjustments for each insulin injection. The system compared these dose adjustments with patient-calculated dose adjustments, using contingency tables and related indicators. We traced the fluctuations of indicators over time, and showed their significance by use of a weighted linear regression model.

By using indicators for measuring patients' adherence to guidelines, this study extends the method comparing insulin dose adjustments to computer-based insulin dose adjustment algorithms [13]. The implementation of insulin adjustment algorithms in a computer program and comparing generated results with patient-calculated results is already documented in another study, but without the use of indicators. Its authors reported an overall rate of adherence to the guideline of $89 \%$. This adherence

Table 3: Indicator rates for each insulin injection.

\begin{tabular}{lcccccc}
\hline Injection & AA & RA & ED & UT & OT & N \\
\hline Lispro before breakfast & 38 & 35 & 2 & 28 & 34 & 426 \\
\hline Lispro before lunch & 37 & 36 & 2 & 33 & 30 & 419 \\
\hline Lispro before dinner & 33 & 45 & 1 & 26 & 41 & 391 \\
\hline Glargine before sleep & 70 & 6 & 2 & 15 & 15 & 420 \\
\hline
\end{tabular}

$\mathrm{N}$ : number of injections. rate is comparable to the AA plus RA ratio (75\%) obtained in our study. They did not deal with day-to-day changes in the adherence, or with particular types of patient behavior, such as under- or over-treatment [14].

We used a locked database in retrospective "offline" settings, but the same method with no change is actually applicable for an online database to monitor patients' adherence in real-time.

Three indicators, namely AA, OT, and UT, are based on statistically known concepts of on- and off-diagonal values of contingency tables. We designed two more indicators of RA and ED, which overlap with other indicators, for their clinical interest. The width of the zones defining these indicators on the contingency table depends on how precise the users of the method want to define their discrete categories. For example, if categories of dose adjustment were defined in two-unit intervals rather than oneunit intervals, the zone of relative agreement would be absorbed by that of absolute agreement (in this case, the categories would be $<-2,-2,0,+2,>+2$, and the contingency table would have 25 cells instead of 49 ).

In our study, patient-calculated dose adjustments were in "absolute agreement" with those generated by the computer in less than half of injections $(\mathrm{AA}=45 \%)$. We introduced the notion of "relative agreement" to detect those injections $(\mathrm{RA}=30 \%)$ which are not strictly the same doses as calculated by the computer, but can be considered acceptable with a degree of tolerance of one unit. A similar strategy is used in the analysis of glucometer per- 
Table 4: Indicator rates for individual patients.

\begin{tabular}{|c|c|c|c|c|c|c|}
\hline Patient number & AA & RA & ED & UT & OT & $\mathbf{N}$ \\
\hline I & 29 & 24 & 8 & 35 & 36 & 66 \\
\hline 2 & 47 & 26 & 3 & 28 & 26 & 58 \\
\hline 3 & 57 & 26 & 0 & 10 & 33 & 42 \\
\hline 4 & 54 & 29 & 0 & 29 & 17 & 63 \\
\hline 5 & 29 & 31 & 2 & 27 & 45 & 49 \\
\hline 6 & 61 & 35 & 0 & 18 & 21 & 66 \\
\hline 7 & 38 & 24 & 2 & 40 & 22 & 50 \\
\hline 8 & 45 & 40 & 0 & 21 & 34 & 58 \\
\hline 9 & 41 & 41 & 0 & 41 & 18 & 68 \\
\hline 10 & 38 & 38 & 5 & 29 & 33 & 58 \\
\hline 11 & 44 & 19 & 2 & 24 & 32 & 63 \\
\hline 12 & 42 & 19 & 3 & 22 & 36 & 67 \\
\hline 13 & 56 & 27 & 0 & 25 & 18 & 55 \\
\hline 14 & 52 & 21 & 2 & 24 & 24 & 58 \\
\hline 15 & 52 & 12 & 2 & 21 & 27 & 56 \\
\hline 16 & 50 & 34 & 1 & 24 & 26 & 68 \\
\hline 17 & 41 & 35 & 2 & 33 & 25 & 51 \\
\hline 18 & 50 & 27 & 2 & 20 & 30 & 56 \\
\hline 19 & 45 & 36 & 0 & 23 & 32 & 53 \\
\hline 20 & 39 & 34 & 0 & 37 & 24 & 62 \\
\hline 21 & 46 & 29 & 2 & 25 & 29 & 65 \\
\hline 22 & 33 & 29 & 3 & 18 & 48 & 66 \\
\hline 23 & 62 & 27 & 0 & 14 & 23 & 64 \\
\hline 24 & 40 & 38 & 0 & 27 & 33 & 52 \\
\hline 25 & 37 & 33 & 0 & 37 & 27 & 52 \\
\hline 26 & 31 & 34 & 2 & 17 & 52 & 64 \\
\hline 27 & 48 & 34 & 0 & 23 & 28 & 64 \\
\hline 28 & 44 & 37 & 3 & 26 & 31 & 62 \\
\hline All & 45 & 30 & 2 & 26 & 30 & 1656 \\
\hline
\end{tabular}

$\mathrm{N}$ : number of injections. formances in form of the well-known Error Grid Analysis $[9,15]$.

While measuring the impact of educational camps, it is especially interesting to know if there is an improvement in patients' adherence during the camp. In our study, this is confirmed by the crescendo curve of cumulative AA and RA indicators during the camp, which is demonstrated by a well-fitted linear model. However, for other indicators we could not fit a statistically significant model, which means that their predictive value for the studied population was limited.

The detection of alarming situations is of paramount importance in monitoring of patient's adherence. The extreme disagreement (ED) indicator is designed to satisfy this need. Although its overall trend is shown to be stable by the regression line, its peaks may necessitate intervention in a real-time monitoring system (figure 3 ).

Two indicators, UT and OT, explain at least partly what patients do when they do not follow the guideline, and why. For example, the frequency of OT is particularly high for a patient (table 4). This implies that this patient tends to try to avoid hyperglycemia by injecting a greater dose of insulin than what is recommended by the guideline. In a monitoring system using our indicators, this patient can be easily detected.

A limitation of our study is that the significance of our indicators depends on the number of observations. Therefore, we need an initial period of a few days before being able to monitor a patient's behavior. Another limitation is the guideline itself. In fact, in 2006, AJD used the same guideline for both patients under insulin lispro and patients under insulin aspart. As our study was non-interventional, we used the guideline which was actually taught to the studied population during 2006. However, the study on this guideline showed some of its shortcomings, and helped developing a new version of the guideline the following years.

Our proposed method and indicators are not specific to insulin self-administration. They can be applied in any other disease where patients decide for their dose of therapy, such as asthma or migraine headache.

\section{Conclusion}

Our method allowed the analysis and monitoring of patients' adherence to an insulin adjustment guideline. The indicators were capable of providing quantitative data on the adherence of patients to the guideline not only for the studied population as a whole, but also for each individual patient. We are currently implementing these indicators in a telemedicine project, supported by the French 


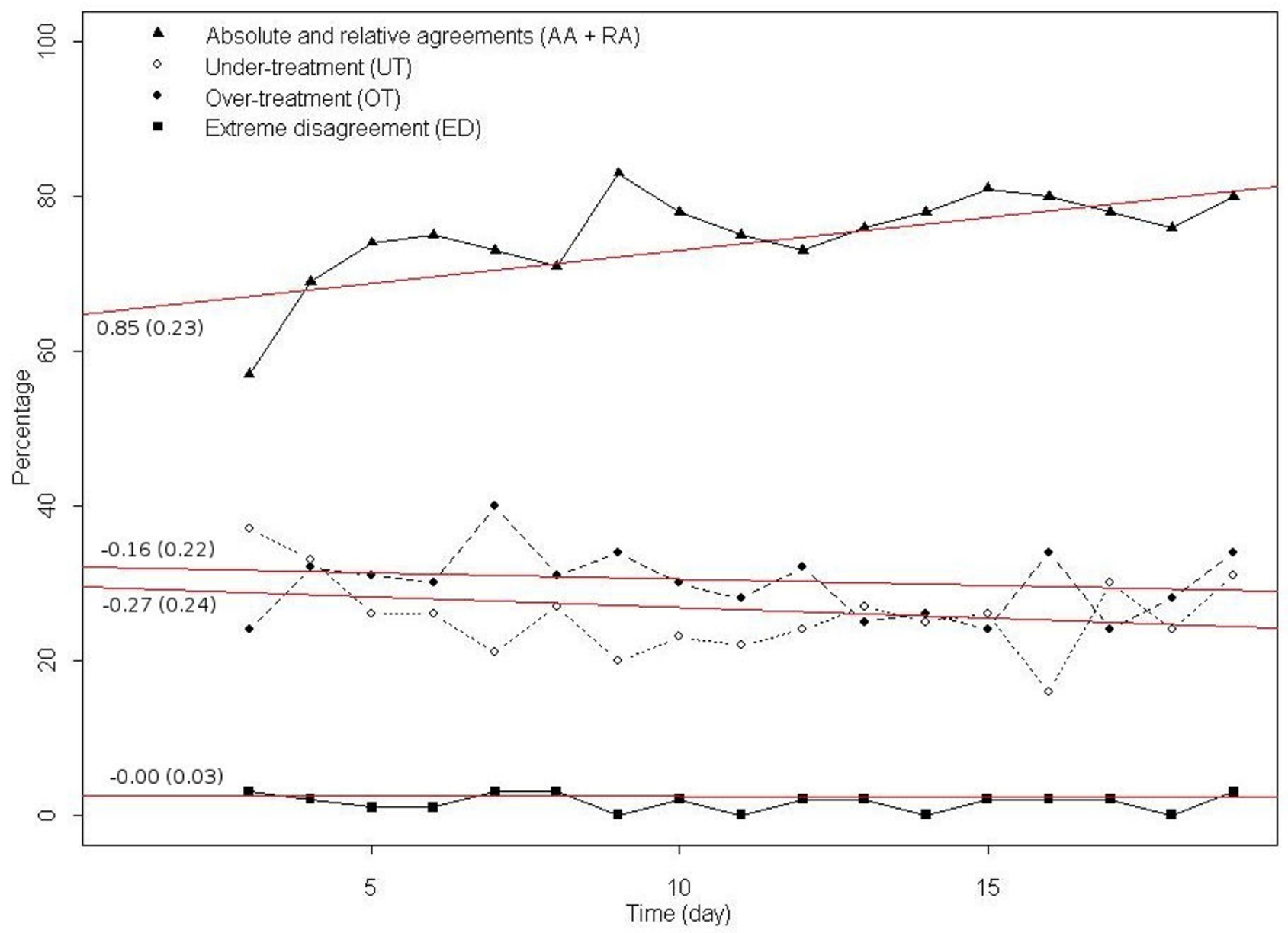

Figure 3

Changes of indicators over time. Coefficients: slope (on the left end of regression lines), standard errors (in parentheses).

National Authority for Health (FNAH), which aimed at monitoring young diabetic patients in real-time, and at alerting their non-adherent behavior to their physicians.

\section{Abbreviations}

CBG: capillary blood glucose; FNAH: French National Authority for Health; AJD: Aide aux Jeunes Diabétique; SD: Standard deviation; CI: Confidence interval; AA: Absolute agreement; RA: Relative agreement; OT: Over treatment; UT: Under treatment; ED: Extreme disagreement.

\section{Competing interests}

The authors declare that they have no competing interests.

\section{Authors' contributions}

MT carried out computer methods, participated in data collection and preparation, participated in the design of indicators and their calculation, and drafted the manuscript. CC carried out the follow-up of patients, obtained authorization from ethics committee, and participated in data collection, preparation and validation. GR verified the clinical value of findings and delimited indicators based on their clinical interest. MC organized patients' observations in camps, and participated in clinical validation of findings. $\mathrm{AB}$ carried out statistical methods, participated in the design of indicators, and verified statistical findings. AV conceived of the study, and participated in its design and coordination and helped to draft the manuscript. All authors read and approved the final manuscript. 


\section{Additional material}

\section{Additional file 1}

Studied guidelines and system source code. This is an html folder containing the studied guidelines in English and French, the de-identified patient database and the source code of the computer system. The file may be downloaded and unzipped in a folder. All the content of the folder can be accessed by double-clicking on "index.htm" file in the folder root. Click here for file

[http://www.biomedcentral.com/content/supplementary/14726947-8-55-S1.zip]

\section{References}

I. Morisky DE, Green LW, Levine DM: Concurrent and predictive validity of a self-reported measure of medication adherence. Med Care 1986, 24:67-74.

2. Norell SE: Accuracy of patient interviews and estimates by clinical staff in determining medication compliance. Soc Sci Med [E] | 98I, I 5:57-6I.

3. Vitolins MZ, Rand CS, Rapp SR, Ribisl PM, Sevick MA: Measuring adherence to behavioral and medical interventions. Control Clin Trials 2000, 2 I(5 Suppl): I88S-94S.

4. Pladevall M, Williams LK, Potts LA, Divine G, Xi H, Lafata JE: Clinical outcomes and adherence to medications measured by claims data in patients with diabetes. Diabetes Care 2004, 27(12):2800-5.

5. Donahoo WT, Bessesen DH, Higbee DR, Lei S, Grunwald GK, Higgins JA: lithium concentration can be used to assess dietary compliance in adults. J Nutr 2004, 134:3133-6.

6. Farmer KC: Methods for measuring and monitoring medication regimen adherence in clinical trials and clinical practice. Clin Ther 1999, 21: 1074-90.

7. Lee WC, Balu S, Cobden D, Joshi AV, Pashos CL: Prevalence and economic consequences of medication adherence in diabetes: a systematic literature review. Manag Care Interface 2006, 19:3I-4I.

8. Wood JR, Laffel LM: Technology and intensive management in youth with type I diabetes: state of the art. Curr Diab Rep 2007, 7:104-13.

9. Albisser AM, Sakkal S, Wright C: Home blood glucose prediction: validation, safety, and efficacy testing in clinical diabetes. Diabetes Technol Ther 2005, 7(3):487-96.

10. Diabcarnet electronic logbook (in French) [http://www.diab carnet.com/]

II. Ihaka R, Gentleman RR: A Language for Data Analysis and Graphics. Journal of Computational and Graphical Statistics 1996, 3:299-3।4

12. Dobson AJ: An introduction to generalized linear models. London: Chapman and Hall; 1990.

13. Choleau C, Albisser AM, Bar-Hen A, Bihan H, Campinos C, Gherbi Z, Jomaa R, Aich M, Cohen R, Reach G: A Novel Method for Assessing Insulin Dose Adjustments by Diabetic Patients. J Diabetes Sci Technol 2007, I(I):3-7.

14. Boukhors Y, Rabasa-Lhoret R, Langelier H, Soultan M, Lacroix A, Chiasson JL: The use of information technology for the management of intensive insulin therapy in type I diabetes mellitus. Diabetes Metab 2003, 29:619-27.

15. Clarke WL, Cox D, Gonder-Frederick LA, Carter W, Pohl SL: Evaluating clinical accuracy of systems for self-monitoring of blood glucose. Diabetes Care 1987, 10:622-8.

\section{Pre-publication history}

The pre-publication history for this paper can be accessed here:

http://www.biomedcentral.com/1472-6947/8/55/prepub

Publish with Biomed Central and every scientist can read your work free of charge

"BioMed Central will be the most significant development for disseminating the results of biomedical research in our lifetime. "

Sir Paul Nurse, Cancer Research UK

Your research papers will be:

- available free of charge to the entire biomedical community

- peer reviewed and published immediately upon acceptance

- cited in PubMed and archived on PubMed Central

- yours - you keep the copyright 